\title{
Influência dos Afloramentos Rochosos sobre a Comunidade Lenhosa no Cerrado stricto sensu
}

\author{
Sílvia da Luz Lima Mota ${ }^{1}$, Israel Marinho Pereira ${ }^{2}$, \\ Evandro Luiz Mendonça Machado ${ }^{2}$, Marcio Leles Romarco de Oliveira ${ }^{2}$, \\ Josiane Silva Bruzinga ${ }^{1}$, Múcio Magno Melo Farnezi², \\ Milton Serpa Meira Junior ${ }^{1}$
}

${ }^{1}$ Departamento de Engenharia Florestal, Universidade de Brasília - UnB, Brasília/DF, Brasil

${ }^{2}$ Departamento de Engenharia Florestal, Universidade Federal dos Vales do Jequitinhonha e Mucuri - UFVJM, Diamantina/MG, Brasil

\begin{abstract}
RESUMO
Objetivou-se verificar como os afloramentos rochosos influenciam os padrões ecológicos das espécies lenhosas no cerrado stricto sensu e se existem espécies indicadoras de ambientes. Foram amostradas dez parcelas e registrados todos os indivíduos lenhosos vivos com DAS $\geq 5 \mathrm{~cm}$ e também as porcentagens de coberturas de afloramentos rochosos. Para avaliar as relações florísticas, foi empregada uma Análise de Correspondência Retificada (DCA), e utilizou-se a Análise de Espécies Indicadoras (ISA), na determinação das preferências das espécies pelos ambientes. Foram calculados os parâmetros fitossociológicos e realizado um teste $t$ de Student para amostras independentes com algumas variáveis estruturais. A DCA confirmou a existência de dois ambientes e mostrou que a presença dos afloramentos rochosos modifica a composição florística entre eles. A ISA mostrou que existem espécies indicadoras de ambientes. A presença dos afloramentos rochosos alterou o conjunto de espécies de maior Valor de Importância (VI) em cada ambiente, porém não houve diferença significativa entre os parâmetros estruturais avaliados em cada ambiente.
\end{abstract}

Palavras-chave: relação substrato-vegetação, ambientes rupestres, espécies indicadoras.

\section{The Influence of Rocky Outcrops on the Woody Community of a Cerrado stricto sensu}

\begin{abstract}
The aim of this study was to verify the presence of environmental indicator species and the influence of rocky outcrops on the ecological patterns of woody plant species in a Cerrado stricto sensu. Ten plots were sampled where information regarding rocky outcrops and all live individuals with DAG $\geq 5 \mathrm{~cm}$ were registered. Detrended Correspondence Analysis (DCA) was performed to assess floristic relations, while Indicator Species Analysis (ISA) was used to determine the environmental preferences of species. Phytosociological parameters were calculated, and the Student's $t$ test was conducted for independent samples with some structural variables. The DAG of live individuals confirmed the existence of two environments and showed that the presence of rocky outcrops changes the floristic composition between them. The ISA verified the presence of environmental indicator species. The presence of rocky outcrops changed the group of species presenting higher Importance Value (VI) in each environment; however, no significant differences were found between the structural parameters assessed in each environment.
\end{abstract}

Keywords: substrate-vegetation relationship, rock environments, indicator species. 


\section{INTRODUÇÃO}

O Cerrado é o segundo maior bioma em extensão territorial no Brasil e possui mais de 200 milhões de hectares, o que corresponde a aproximadamente 23\% da área total do território nacional (Ribeiro \& Walter, 2008). Por ocupar área muito extensa, o Cerrado, segundo Ribeiro \& Walter (2008), ocorre em altitudes bastante variadas, de $300 \mathrm{~m}$ a mais de $1.600 \mathrm{~m}$; estas grandes amplitudes espaciais e de altitude promovem uma grande heterogeneidade ambiental no Cerrado (Felfili et al., 2004), refletindo em diversificado mosaico vegetacional ao longo do bioma.

Ribeiro \& Walter (2008) apontam 11 tipos fitofisionômicos, divididos entre formações florestais, savânicas e campestres; dentre estes, o cerrado stricto sensu é o mais extenso, ocupando cerca de 70\% do bioma (Felfili \& Silva-Junior, 2005). Esse padrão acarreta maior diversidade de subtipos, com ambientes de vegetação densa, típica e rala sobre solos profundos e ambientes rupestres, com solos rasos e afloramentos rochosos, e com a presença de arbustos e árvores (Ribeiro \& Walter, 2008).

Apesar de os ambientes rupestres ocuparem apenas cerca de 7\% do bioma Cerrado (Reatto et al., 2008), em geral estes apresentam alta diversidade de espécies arbustivo-arbóreas e densidade variável (Pinto et al., 2009; Moura et al., 2010; Lima et al., 2010), dependendo do volume de solo (Ribeiro \& Walter, 2008). Observa-se nestes ambientes a presença de flora esclerófila adaptada a condições extremas, como elevada insolação e solos rasos, com baixa umidade e naturalmente pobres (Amaral et al., 2006).

Diversos fatores bióticos e abióticos, bem como suas interações, são citados como os principais responsáveis pela distribuição espacial de espécies arbustivo-arbóreas (Kent \& Coker, 1992). Segundo Haridasan (2000), variações na fertilidade e nas propriedades físicas dos solos refletem em variações da composição florística e de estrutura e distribuição de espécies. Felfili et al. (2004), em um estudo sobre fitogeografia, compararam a composição florística e a estrutura da vegetação lenhosa do cerrado stricto $s e n s u$, e seus resultados mostram um gradiente florístico e estrutural, seguindo a variação ambiental, especialmente a profundidade do solo.

Existem muitos estudos envolvendo o bioma Cerrado, sendo a maioria realizada em áreas com relevo plano a suave ondulado e localizadas nos planaltos sobre solos profundos (Ratter et al., 2003). Porém, pouco se sabe sobre a correlação entre a cobertura de rochas e as informações florísticas e estruturais da vegetação lenhosa em ambientes rupestres no Cerrado (Scarano, 2007; Abreu et al., 2012).

Diante da necessidade de pesquisas sobre a vegetação lenhosa de ambientes rupestres no Cerrado e para subsidiar projetos de conservação e recuperação desta fisionomia, este estudo tem como objetivo verificar como os afloramentos rochosos influenciam os padrões da composição florística e a estrutura fisionômica das espécies lenhosas, além de constatar se existem espécies indicadoras de um tipo específico de ambiente.

\section{MATERIAL E MÉTODOS}

A área de estudo está situada no Parque Estadual do Biribiri (PEB), em Diamantina-MG. O regime climático desta região é tipicamente tropical, $\mathrm{Cwb}$ na classificação de Koppen, com precipitação média anual de 1.250 a $1.550 \mathrm{~mm}$, temperatura na faixa de $18^{\circ} \mathrm{C}$ a $19^{\circ} \mathrm{C}$, umidade relativa em média de $75,6 \%$ e altitude acima dos 900 metros. As rochas quartzíticas, muito características e presentes na região, são compostas quase que exclusivamente do mineral quartzo, o que promove a formação de solos arenosos e rasos (Neves et al., 2005). O inventário foi realizado em um trecho de 5,3 ha de cerrado stricto sensu dentro do $\mathrm{PEB}$, localizado na coordenada

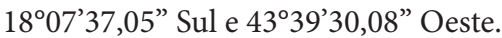

Para amostrar a vegetação arbustivo-arbórea, foram demarcadas de forma sistemática dez parcelas de $20 \times 50 \mathrm{~m}\left(1000 \mathrm{~m}^{2}\right)$, com espaçamento de $50 \mathrm{~m}$ entre estas, totalizando uma área amostral de 1 ha, ao longo do transecto de 550 metros, conforme Figura 1.

Em cada parcela, foram registrados todos os indivíduos arbustivo-arbóreos vivos que apresentaram fuste com diâmetro a $0,30 \mathrm{~m}$ do solo $(D A S) \geq 5,0 \mathrm{~cm}$, os quais foram marcados com 


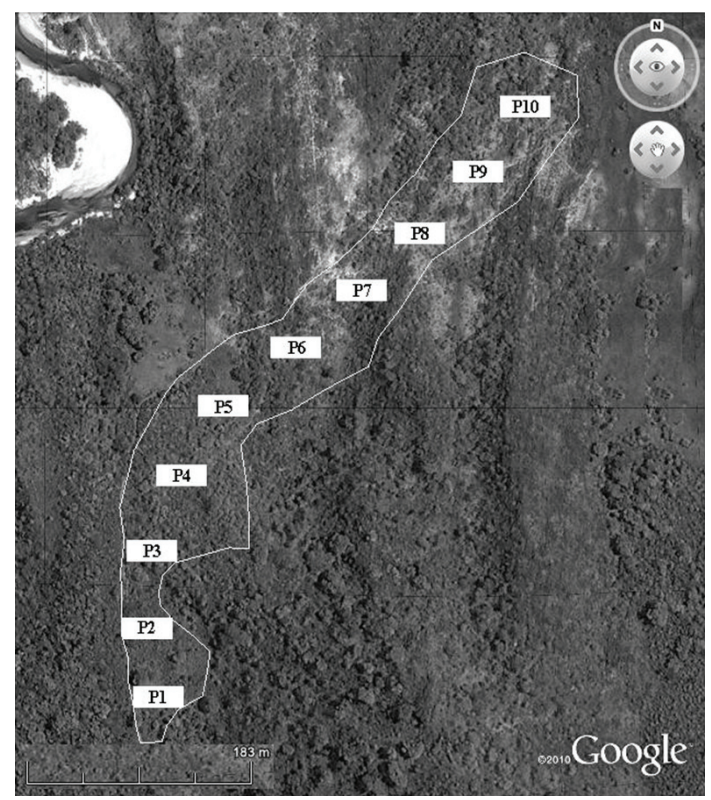

Figura 1. Delimitação da área de estudo e distribuição das parcelas no trecho de cerrado stricto sensu no PEB. Figure 1. Study area delimiting and distribution of plots within the cerrado stricto sensu stretch in PEB.

etiquetas de alumínio numeradas, tiveram suas alturas totais (HT) mensuradas com uma régua graduada e DAS medido com suta dendrométrica. Os indivíduos bifurcados ou com caules múltiplos foram medidos quando a raiz da soma dos quadrados dos seus diâmetros era $\geq 5,0 \mathrm{~cm}$, por meio da fórmula DASf $=\sqrt{ }_{\mathrm{DAS}_{1}}{ }^{2}+\mathrm{DAS}_{2}{ }^{2}+\ldots+\mathrm{DAS}_{\mathrm{n}}{ }^{2}$. Com base nesta fórmula, no momento das análises, indivíduos bifurcados tiveram seus DAS fundidos (DASf), sendo considerados como um único indivíduo; destes, foi considerada apenas a altura total do maior fuste.

As espécies foram classificadas nas famílias reconhecidas pelo sistema do Angiosperm Phylogeny Group III (APG III, 2009) e o material botânico testemunho encontra-se depositado no Herbário Dendrológico Jeanine Felfili (HDJF) da Universidade Federal dos Vales do Jequitinhonha e Mucuri (UFVJM).

Foi avaliada a cobertura de rochas em cada parcela, sendo essa avaliação feita de forma visual, sempre pelo mesmo observador, e adaptada da escala de valores de Braun-Blanquet (MuellerDombois \& Ellenberg, 1974). As notas atribuídas aos afloramentos variaram de zero a quatro, de acordo com sua frequência em cada parcela, sendo zero na ausência do mesmo; um: 1-25\% de ocorrência na parcela; dois: $26-50 \%$ de ocorrência; três: 51-75\%, e quatro: 76-100\%. De acordo com essa escala, em um caminhamento por toda a área, o observador verificou qual a porcentagem de afloramentos rochosos cobre cada parcela, sendo que o valor da nota dada a cada parcela foi usada como variável categórica na matriz utilizada nas análises multivariadas.

Analisando-se as relações florísticas entre as parcelas, para verificar o grau de semelhança entre estas e se as mesmas formam grupos distintos, foi empregada uma técnica de análise multivariada, a análise de correspondência retificada, do inglês Detrended Correspondence Analysis (DCA). Esta é uma técnica de estatística não paramétrica que produz um diagrama de ordenação no qual as parcelas se distribuem de acordo com a maior ou menor similaridade entre si (ter Braak, 1987). A DCA foi realizada com base na presença e na ausência de espécies (variáveis qualitativas), e de afloramentos rochosos (variáveis categóricas), nas dez parcelas; foi realizada no programa PC-ORD for Windows versão 4.0 (McCune \& Mefford, 1999).

Foi utilizada a análise de espécies indicadoras (Dufrêne \& Legendre, 1997) no programa PC-ORD for Windows versão 4.0 (McCune \& Mefford, 1999) como ferramenta na determinação das preferências das espécies pelos ambientes. Este método combina as informações de concentração da abundância de uma espécie em certo grupo e sua fidelidade de ocorrência a este mesmo grupo. Um valor indicador (VI) é gerado para cada espécie em cada grupo e a significância da diferença de um valor gerado pelo acaso é determinada pelo teste de permutação de Monte Carlo. Com isso, uma espécie só é considerada indicadora de um ambiente quando apresenta o maior VI para o mesmo e o resultado do teste de Monte Carlo é significativo.

Para descrever a estrutura horizontal de cada ambiente formado pelo agrupamento das parcelas, foram calculados, por espécie, os parâmetros fitossociológicos clássicos propostos por MuellerDombois \& Ellenberg (1974): densidade, frequência e dominância expressa pela área basal por hectare com seus valores relativos.

Foi realizado um teste $t$ de Student para amostras independentes com as variáveis número 
de indivíduos, densidade, área basal, diâmetro médio e altura total média, realizado no programa R 2.15.1 (R Core Team, 2012), para verificar se existe diferença significativa na estrutura da comunidade lenhosa nos dois ambientes.

\section{RESULTADOS E DISCUSSÃO}

O levantamento da cobertura de rochas quartzíticas nas parcelas aponta para a formação de dois ambientes distintos. As parcelas 1, 2, 3 e 4 formam o ambiente 1, o qual não apresenta afloramentos rochosos dentro das quatro parcelas; estas apresentam substrato visualmente homogêneo, profundo e úmido por estarem em uma depressão entre os afloramentos rochosos, que são presentes em quase todo o fragmento de cerrado stricto sensu. Portanto, toda a área de cada parcela $\left(1.000 \mathrm{~m}^{2}\right)$, neste ambiente, está disponível para o desenvolvimento das espécies lenhosas.

Já as parcelas 5, 6, 7, 8, 9 e 10 formam o ambiente 2 e apresentam cobertura de afloramentos rochosos acima de $20 \%$ de sua área total, sendo estas coberturas de $20 \%, 25 \%, 25 \%, 45 \%, 45 \%$ e $40 \%$, respectivamente. Neste ambiente, existe uma diversidade de substratos, que variam de acordo com a maior ou menor cobertura de afloramentos. Visualmente, a parcela 5 é bastante semelhante às parcelas do ambiente 1 , porém apresenta um pequeno afloramento rochoso concentrado em um dos seus extremos, além dos $25 \%$ de cobertura por afloramentos rochosos; o solo presente nas parcelas 6 e 7 é arenoso e, consequentemente, tem baixa capacidade de retenção de água e nutrientes, e as parcelas 8,9 e 10 têm afloramentos rochosos extensos, que cobrem quase a metade de suas áreas, reduzindo bastante a área útil para o desenvolvimentos das espécies lenhosas.

A presença dos afloramentos rochosos dentro de algumas parcelas influencia na diferenciação da composição florística entre os dois ambientes e esta pode ser observada na DCA (Figura 2). O autovalor obtido para o eixo 1 da ordenação foi alto $(>0,5)$; este eixo forma um gradiente longo, o que garante que existe substituição de espécies entre seus extremos (ter Braak, 1995), ou seja, entre os ambientes sem (1) e com (2) afloramentos rochosos. Já o eixo 2 da DCA é curto, com autovalor $(<0,5)$ (ter Braak, 1995); assim, pode-se perceber que, dentro de cada ambiente, a composição florística das parcelas é semelhante.

A presença e a ausência dos afloramentos rochosos nas parcelas parecem não afetar as famílias botânicas mais bem sucedidas em relação à riqueza, pois Fabaceae e Vochysiaceae foram as que apresentaram maior número de espécies nos

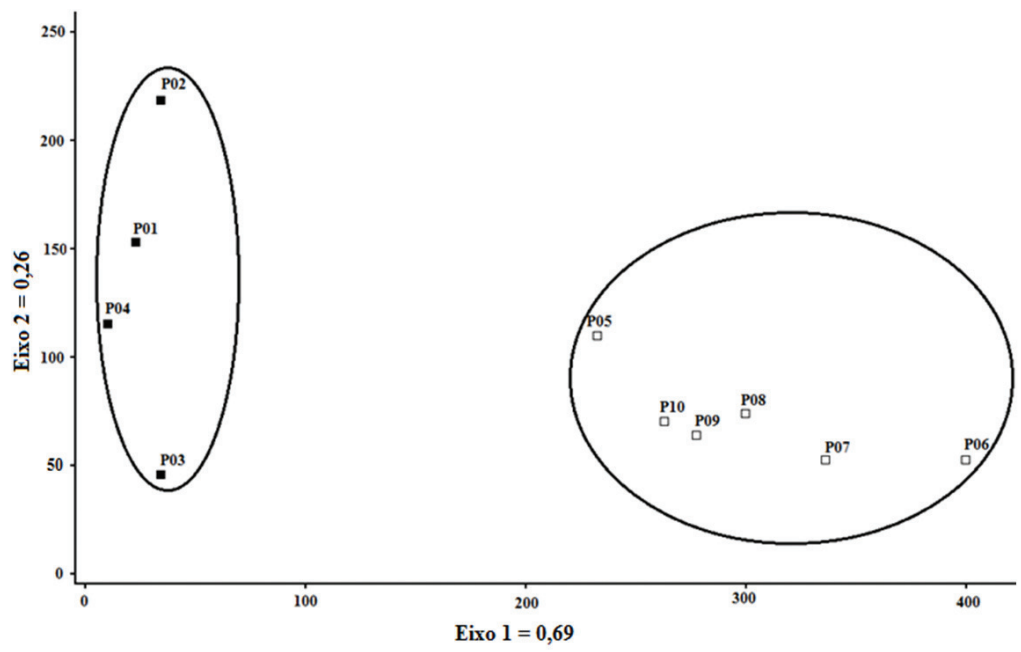

Figura 2. Diagrama de ordenação da análise de correspondência retificada (DCA) da composição florística (presença e ausência de espécies) das dez parcelas no trecho de cerrado stricto sensu em Diamantina-MG. $\mathbf{a}=$ parcelas sem afloramentos rochosos; $\square=$ parcelas com afloramentos rochosos.

Figure 2. Ordination analysis of correspondence linearity diagram (DCA) of the vegetation composition (presence and absence of species) for the ten plots in the area of stricto sensu savanna at Diamantina, MG. Where: = plots no rock outcrops and $\square=$ plots with rock outcrops. 
dois ambientes. No ambiente 1, a família Fabaceae foi representada por dez espécies e Vochysiaceae, por quatro; este padrão se inverte no ambiente 2, no qual Vochysiaceae parece ser menos influenciada pela presença dos afloramentos rochosos; a mesma apresenta seis espécies neste ambiente e Fabaceae, apenas quatro espécies.

Estas duas famílias são sempre mencionadas como mais ricas em estudos de cerrado relacionados ao bioma Cerrado em ambientes rupestres, conforme relatam Amaral et al. (2006), em BrasíliaDF; Miranda et al. (2007), em Serra Dourada-GO; Pinto et al. (2009), em Cocalzinho de Goiás-GO, e Moura et al. (2010), em Goiás. De acordo com Goodland (1979), a superioridade da família Fabaceae no bioma Cerrado deve-se ao fato de a maioria de suas espécies apresentar capacidade de fixação biológica de nitrogênio (FBN). Já com relação ao sucesso da família Vochysiaceae, Haridasan (2000) destaca que várias de suas espécies são acumuladoras de alumínio. Estes dois autores, portanto, afirmam que espécies com capacidade de FBN e acumuladoras de alumínio possuem vantagem competitiva ou adaptativa sobre as demais.

O agrupamento das parcelas observado na DCA corrobora com a separação dos dois ambientes verificada na análise da cobertura por afloramentos rochosos e mostra a dissimilaridade florística existente entre eles. Essa diferença na composição florística ocorre porque existe um numeroso grupo de espécies exclusivas de cada ambiente.
Das 58 espécies encontradas na amostragem, 22 são exclusivas do ambiente 1 e 19 são exclusivas do ambiente 2, restando apenas 17 espécies comuns aos dois.

Embora existam muitas espécies exclusivas de cada ambiente, poucas destas são indicadoras do seu ambiente. Do total de espécies amostradas, apenas sete $(12,07 \%)$ apresentaram preferência significativa (fidelidade e abundância) por um dos ambientes, de acordo com a análise de espécies indicadoras (ISA), sendo quatro $(6,90 \%)$ pelo ambiente 1 (sem afloramento rochoso) e três (5,17\%) pelo ambiente 2 (com afloramento rochoso) (Tabela 1).

Segundo os autores Silva-Junior $(2005,2012)$, Silva-Junior \& Pereira (2009) e Lorenzi (1992, 1998, 2002), Bowdichia virgilioides, Dalbergia miscolobium e Terminalia fagifolia ocorrem em cerrado stricto sensu, cerradão e matas secas, sendo que a última espécie pode ocorrer também nas bordas de matas de galeria. Já o Astronium graveolens ocorre nas formações florestais do Complexo Atlântico e nas matas de planalto do interior do País. Estas quatro espécies indicadoras do ambiente 1 apresentam grande porte, com altura que pode alcançar entre 8 e $25 \mathrm{~m}$, e troncos que variam de $30 \mathrm{a} 60 \mathrm{~cm}$ de diâmetro; possuem madeiras densas $\left(0,81 \mathrm{~g} \cdot \mathrm{cm}^{-3}\right.$ a $1,0{\left.\mathrm{~g} . \mathrm{cm}^{-3}\right)}^{-3}$ e de grande durabilidade, com alto valor comercial devido à sua ampla utilização. As parcelas que representam o ambiente 1, embora estejam em um ambiente rupestre, ocorrem nas depressões entre os afloramentos rochosos e apresentam

Tabela 1. Espécies com resultados significativos na análise de espécie indicadora (ISA) realizada com base nos dois ambientes em relação aos afloramentos rochosos. VIO = valor indicador observado; VIE = valor indicador esperado; $\mathrm{s}=$ desvio padrão; $\mathrm{p}=$ significância, ${ }^{\star} \mathrm{p}<0,05 ;{ }^{* *} \mathrm{p}<0,01$.

Table 1. Species with significative results in the indicator species analysis (ISA) which was done basing on the two ambient in relation with the rock outcrops. VIO = observed indicator value; VIE = expected indicator value; $\mathrm{s}=$ standard deviation; $\mathrm{p}=$ significance, ${ }^{\star} \mathrm{p}<0.05 ;{ }^{*} \mathrm{p}<0.01$.

\begin{tabular}{|c|c|c|c|c|}
\hline Ambientes & \multirow{2}{*}{ VIO } & \multicolumn{3}{|c|}{ VIE } \\
\hline Espécies & & Média & $\mathbf{s}$ & p \\
\hline \multicolumn{5}{|l|}{ Sem afloramento rochoso } \\
\hline Astronium graveolens & 75,0 & 31,5 & 14,9 & * \\
\hline Bowdichia virgilioides & 83,3 & 46,9 & 14,2 & * \\
\hline Dalbergia miscolobium & 86,8 & 50,9 & 13,3 & * \\
\hline Terminalia fagifolia & 72,8 & 37,8 & 15,1 & * \\
\hline \multicolumn{5}{|l|}{ Com afloramento rochoso } \\
\hline Eremanthus erythropappus & 93,6 & 51,4 & 13,3 & * \\
\hline Kielmeyera lathrophyton & 100,0 & 50,7 & 15,9 & ** \\
\hline Kielmeyera rubriflora & 83,3 & 43,0 & 15,2 & * \\
\hline
\end{tabular}


substrato mais profundo, maior deposição de matéria orgânica e maior umidade do que as demais parcelas amostradas, justificando a preferência e o sucesso de estabelecimento das suas quatro espécies indicadoras.

Ao contrário, as três espécies indicadoras do ambiente 2 são adaptadas a ambientes pobres em nutrientes e solos rasos, e mostram-se típicas de áreas abertas, como os campos de altitude e o cerrado rupestre (Lorenzi, 2002; Fujaco et al., 2006). Kielmeyera lathrophyton apresenta porte médio, altura entre 8 e $14 \mathrm{~m}$, diâmetro de 30 a $50 \mathrm{~cm}$, madeira leve, macia, pouco resistente, pouco durável, de 0,67 g.cm ${ }^{-3}$ e de uso restrito (Lorenzi, 2002). Kielmeyera rubriflora apresenta características semelhantes à $K$. lathrophyton, porém com indivíduos de pequeno porte, entre 4 e 5 metros de altura (Lorenzi, 2002). Eremanthus erythropappus é uma árvore de porte médio, sua altura varia de 2 a $10 \mathrm{me}$ o diâmetro pode atingir até $35 \mathrm{~cm}$, desenvolvendo-se em solos pouco férteis, rasos e predominantemente em áreas de campos com altitude entre $1.000 \mathrm{e}$ $1.700 \mathrm{~m}$ (Scolforo et al., 2002). As populações do gênero Eremanthus spp. encontram-se distribuídas preferencialmente em locais onde ocorreram eventos de natureza antrópica, em regiões queimadas ou litológicas cujos solos são rasos e com poucos nutrientes, como é o caso dos solos originados dos quartzitos e das cangas lateríticas (Fujaco et al., 2006).

Foram amostrados, no total, 445 indivíduos lenhosos distribuídos em 58 espécies, 43 gêneros e 28 famílias botânicas (Tabela 2).

Um pequeno grupo de espécies ( 7 no ambiente 1 e 6 no ambiente 2) detém mais de $50 \%$ da estrutura em cada ambiente, sendo as espécies que obtiveram maior sucesso de ocupação e estabelecimento diante dos recursos oferecidos em cada ambiente. Portanto, estas são as espécies que dominam a vegetação $\mathrm{e}$ devem ser prioritárias nas ações de conservação e recuperação de ambientes semelhantes. A lista de espécies com maior VI nos dois ambientes confirma a dissimilaridade florística existente entre os mesmos, vista na DCA, e apoia a análise de espécies indicadoras, segundo a qual cada ambiente possui um conjunto de espécies distinto e com características ecológicas diferentes.
No ambiente 1, as espécies com maior VI foram Terminalia fagifolia (13,27\%), Dalbergia miscolobium (9,23\%), Lafoensia pacari (9,16\%), Qualea multiflora (6,16\%), Bowdichia virgilioides (4,90\%), Hymenaea stigonocarpa $(4,88 \%)$ e Platypodium elegans $(4,48 \%)$.

Terminalia fagifolia, Dalbergia miscolobium e Bowdichia virgilioides, além de estarem entre as de maior VI, são também indicadoras do ambiente 1 e suas características ecológicas já foram discutidas anteriormente. As outras quatro espécies de maior VI no ambiente 1, segundo Lorenzi (2002) e SilvaJunior (2012), são de grande porte, com alturas entre 8 e $18 \mathrm{~m}$, e troncos de 35 a $50 \mathrm{~cm}$ de diâmetro. São espécies que se desenvolvem bem em solos profundos e bem estruturados, pois, segundo os mesmos autores, ocorrem geralmente em cerrado stricto sensu, cerradão, matas estacionais e bordas de matas secas e de galeria. Isso pode explicar o sucesso destas no ambiente 1 , onde não existem afloramentos rochosos e, portanto, por estar em uma depressão entre as rochas, apresenta solos mais profundos e úmidos, em comparação ao ambiente 2 .

O conjunto de espécies com maior VI no ambiente 2 é composto por Kielmeyera lathrophyton (15,99\%), Qualea dichotoma (9,38\%), Kielmeyera rubriflora (9,17\%), Aspidosperma cylindrocarpon (7,38\%), Eremanthus erythropappus (7,18\%) e Norantea adamantium (6,59\%).

Três espécies deste conjunto coincidem com as espécies indicadoras do ambiente 2: Kielmeyera lathrophyton, Kielmeyera rubriflora e Eremanthus erythropappus, cujas características ecológicas foram discutidas anteriormente. Das três restantes, Norantea adamantium, assim como as indicadoras, é uma espécie típica de ambientes rochosos, possui raízes adventícias e seu tronco pode chegar a $30 \mathrm{~cm}$ de diâmetro; ocorre em campo cerrado, campo rupestre e cerrado stricto sensu em terrenos rochosos acima de $900 \mathrm{~m}$ de altitude (Silva-Junior, 2005), como é o caso do ambiente 2 deste trabalho.

Já as espécies Qualea dichotoma e Aspidosperma cylindrocarpon são típicas de formações florestais, sendo que a primeira ocorre geralmente em matas de galeria e florestas estacionais, e pode alcançar $80 \mathrm{~cm}$ de diâmetro (Silva-Junior \& Pereira, 2009) e altura de 10 a 18m (Lorenzi, 1992). A segunda espécie ocorre na floresta semidecídua e pode alcançar altura de 8 a 
Tabela 2. Relação das famílias e espécies arbustivo-arbóreas registradas nos ambientes 1 (sem afloramentos rochosos) e 2 (com afloramentos rochosos) no trecho de cerrado stricto sensu, Diamantina-MG, e seus parâmetros fitossociológicos. $\mathrm{DR}=$ densidade relativa (\%); DoR = dominância relativa (\%); FR = frequência relativa (\%), e VI = valor de importância (\%).

Table 2. Relation of the families with shrubs and arboreal species registered in ambient 1 (no rock outcrops) and 2 (rock outcrops) at the area of rocky savanna, Diamantina, MG, and their phytosociological parameters. Which correspond: $\mathrm{DR}=$ relative density $(\%) ; \mathrm{DoR}=$ relative dominance $(\%) ; \mathrm{FR}=$ relative frequency $(\%)$ and $\mathrm{VI}=$ importance value (\%).

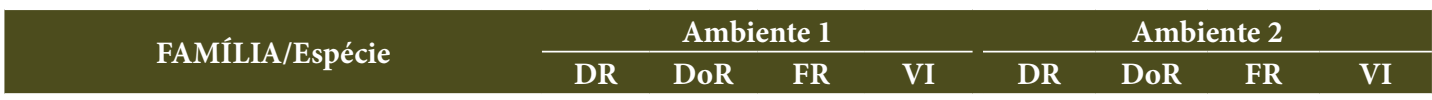

ANACARDIACEAE

Astronium graveolens Jacq

$\begin{array}{llll}3,28 & 0,83 & 4,84 & 2,98\end{array}$

APOCYNACEAE

\begin{tabular}{|c|c|c|c|c|c|c|c|c|}
\hline Aspidosperma cylindrocarpon Müll. Arg. & & & & & 6,49 & 10,64 & 5,00 & 7,38 \\
\hline Aspidosperma tomentosum Mart. & 0,55 & 0,16 & 1,61 & 0,77 & & & & \\
\hline Hancornia speciosa B.A.Gomes & & & & & 0,76 & 0,43 & 2,50 & 1,23 \\
\hline \multicolumn{9}{|l|}{ ASTERACEAE } \\
\hline Eremanthus erythropappus (DC.) MacLeish & 0,55 & 0,15 & 11,6 & 0,77 & 8,4 & 5,66 & 7,5 & 7,18 \\
\hline \multicolumn{9}{|l|}{ BIGNONIACEAE } \\
\hline Handroanthus ochraceus (Cham.) Mattos & & & & & 0,38 & 0,29 & 1,25 & 0,64 \\
\hline Jacaranda micrantha Cham. & 4,37 & 0,99 & 1,61 & 2,33 & & & & \\
\hline \multicolumn{9}{|l|}{ CELASTRACEAE } \\
\hline Plenckia populnea Reissek & 1,09 & 0,55 & 1,61 & 1,09 & 0,38 & 1,24 & 1,25 & 0,96 \\
\hline \multicolumn{9}{|l|}{ CHRYSOBALANACEAE } \\
\hline Hirtella glandulosa Spreng. & & & & & 0,38 & 0,50 & 1,25 & 0,71 \\
\hline \multicolumn{9}{|l|}{ CLUSIACEAE } \\
\hline Kielmeyera lathrophyton Saddi & & & & & 19,47 & 21,01 & 7,50 & 15,99 \\
\hline Kielmeyera rubriflora Cambess. & & & & & 13,36 & 7,91 & 6,25 & 9,17 \\
\hline \multicolumn{9}{|l|}{ COMBRETACEAE } \\
\hline Terminalia argentea (Cambess.) Mart. & 1,09 & 0,32 & 3,23 & 1,55 & & & & \\
\hline Terminalia fagifolia Mart. & 12,02 & 22,96 & 4,84 & 13,2 & 0,38 & 0,24 & 1,25 & 0,62 \\
\hline Terminalia glabrescens Mart. & 4,92 & 5,29 & 1,61 & 3,94 & & & & \\
\hline \multicolumn{9}{|l|}{ DILLENIACEAE } \\
\hline Davilla elliptica A.St.-Hil. & 0,55 & 0,50 & 1,61 & 0,89 & & & & \\
\hline \multicolumn{9}{|l|}{ EBENACEAE } \\
\hline Diospyros burchellii Hiern. & 0,55 & 0,17 & 1,61 & 0,78 & & & & \\
\hline Diospyros sericea A.DC. & 1,09 & 1,26 & 1,61 & 1,32 & & & & \\
\hline \multicolumn{9}{|l|}{ FABACEAE } \\
\hline Bowdichia virgilioides Kunth & 5,46 & 2,77 & 6,45 & 4,90 & 1,15 & 1,88 & 2,50 & 1,84 \\
\hline Copaifera langsdorffii Desf. & 2,19 & 3,99 & 1,61 & 2,60 & 1,15 & 4,60 & 2,50 & 2,75 \\
\hline Dalbergia miscolobium Benth. & 12,02 & 9,21 & 6,45 & 9,23 & 1,91 & 3,94 & 3,75 & 3,20 \\
\hline Hymenaea stigonocarpa Mart. ex Hayne & 4,37 & 7,04 & 3,23 & 4,88 & 0,38 & 0,23 & 1,25 & 0,62 \\
\hline Machaerium opacum Vogel & 1,09 & 1,80 & 1,61 & 1,50 & & & & \\
\hline Machaerium villosum Vogel & 0,55 & 0,29 & 1,61 & 0,82 & & & & \\
\hline Plathymenia reticulata Benth. & 0,55 & 0,34 & 1,61 & 0,83 & & & & \\
\hline Platypodium elegans Vogel & 7,65 & 4,18 & 1,61 & 4,48 & & & & \\
\hline Pterodon emarginatus Vogel & 1,09 & 6,03 & 1,61 & 2,91 & & & & \\
\hline Stryphnodendron adstringens (Mart.) Cov. & 0,55 & 0,19 & 1,61 & 0,78 & & & & \\
\hline \multicolumn{9}{|l|}{ HUMIRIACEAE } \\
\hline Humiria balsamifera Aubl. & & & & & 0,76 & 2,27 & 2,50 & 1,84 \\
\hline \multicolumn{9}{|l|}{ LAURACEAE } \\
\hline Ocotea lancifolia (Schott.) Mez & 0,55 & 0,14 & 1,61 & 0,77 & & & & \\
\hline
\end{tabular}


Tabela 2. Continuação...

Table 2. Continued...

\begin{tabular}{|c|c|c|c|c|c|c|c|c|}
\hline \multirow{2}{*}{ FAMÍLIA/Espécie } & \multicolumn{4}{|c|}{ Ambiente 1} & \multicolumn{4}{|c|}{ Ambiente 2} \\
\hline & DR & DoR & FR & VI & DR & DoR & FR & VI \\
\hline \multicolumn{9}{|l|}{ LYTHRACEAE } \\
\hline Lafoensia pacari Cham. \& Schltdl. & 9,84 & 11,19 & 6,45 & 9,16 & 3,44 & 1,50 & 3,75 & 2,90 \\
\hline Byrsonima basiloba A.Juss. & & & & & 0,38 & 0,05 & 1,25 & 0,56 \\
\hline Byrsonima coccolobifolia Kunth & & & & & 0,76 & 0,36 & 2,50 & 1,21 \\
\hline Byrsonima pachyphylla A.Juss. & 0,55 & 0,24 & 1,61 & 0,80 & & & & \\
\hline \multicolumn{9}{|l|}{ MALVACEAE } \\
\hline Eriotheca pentaphylla (Vell.) A.Robyns & 0,55 & 0,46 & 1,61 & 0,87 & & & & \\
\hline $\begin{array}{l}\text { Pseudobombax longiflorum (Mart. \& Zucc.) } \\
\text { A.Robyns }\end{array}$ & 1,64 & 3,63 & 1,61 & 2,29 & & & & \\
\hline \multicolumn{9}{|l|}{ MARCGRAVIACEAE } \\
\hline Norantea adamantium Cambess. & & & & & 6,49 & 8,28 & 5,00 & 6,59 \\
\hline \multicolumn{9}{|l|}{ MELIACEAE } \\
\hline Cabralea canjerana (Vell.) Mart. & 0,55 & 0,26 & 1,61 & 0,81 & & & & \\
\hline \multicolumn{9}{|l|}{ MYRTACEAE } \\
\hline Myrcia multiflora (Lam.) DC. & 0,55 & 0,21 & 1,61 & 0,79 & 0,38 & 0,18 & 1,25 & 0,61 \\
\hline Myrcia splendens (Sw.) DC. & 0,55 & 0,15 & 1,61 & 0,77 & 0,38 & 0,21 & 1,25 & 0,62 \\
\hline Myrcia tomentosa (Aubl.) DC. & 0,55 & 0,27 & 1,61 & 0,81 & & & & \\
\hline \multicolumn{9}{|l|}{ OCHNACEAE } \\
\hline Ouratea hexasperma (A.St.-Hil.) Baill. & & & & & 3,05 & 0,82 & 3,75 & 2,54 \\
\hline \multicolumn{9}{|l|}{ OPILIACEAE } \\
\hline $\begin{array}{l}\text { Agonandra brasiliensis Miers ex Benth. \& } \\
\text { Hook. }\end{array}$ & & & & & 0,38 & 0,14 & 1,25 & 0,59 \\
\hline \multicolumn{9}{|l|}{ EUPHORBIACEAE } \\
\hline Pera glabrata (Schott) Poepp. ex Baill. & 2,19 & 1,06 & 3,23 & 2,16 & 0,38 & 0,10 & 1,25 & 0,58 \\
\hline \multicolumn{9}{|l|}{ PROTEACEAE } \\
\hline Roupala montana Aubl. & 2,73 & 1,12 & 4,84 & 2,90 & 0,38 & 0,14 & 1,25 & 0,59 \\
\hline \multicolumn{9}{|l|}{ RUBIACEAE } \\
\hline Rubiaceae não identificada 1 & 0,55 & 0,70 & 1,61 & 0,95 & & & & \\
\hline $\begin{array}{l}\text { Tocoyena formosa (Cham. \& Schltdl.) } \\
\text { K.Schum. }\end{array}$ & & & & & 0,76 & 0,13 & 2,50 & 1,13 \\
\hline \multicolumn{9}{|l|}{ SAPOTACEAE } \\
\hline $\begin{array}{l}\text { Chrysophyllum marginatum (Hook. \& Arn.) } \\
\text { Radlk. }\end{array}$ & & & & & 0,38 & 0,31 & 1,25 & 0,65 \\
\hline Pouteria ramiflora (Mart.) Radlk. & & & & & 1,15 & 0,53 & 2,50 & 1,39 \\
\hline Pouteria torta (Mart.) Radlk. & & & & & 0,76 & 0,61 & 2,50 & 1,29 \\
\hline \multicolumn{9}{|l|}{ URTICACEAE } \\
\hline Cecropia pachystachya Trécul & 1,09 & 0,21 & 1,61 & 0,97 & & & & \\
\hline \multicolumn{9}{|l|}{ VOCHYSIACEAE } \\
\hline Callisthene minor Mart. & 0,55 & 0,17 & 1,61 & 0,78 & 3,82 & 3,25 & 3,75 & 3,61 \\
\hline Qualea dichotoma (Mart.) Warm. & 2,19 & 1,24 & 1,61 & 1,68 & 12,60 & 10,55 & 5,00 & 9,38 \\
\hline Qualea grandiflora Mart. & 1,64 & 4,25 & 3,23 & 3,04 & & & & \\
\hline Qualea multiflora Mart. & 7,10 & 4,93 & 6,45 & 6,16 & 2,29 & 2,31 & 3,75 & 2,78 \\
\hline Qualea parviflora Mart. & & & & & 3,44 & 2,20 & 2,50 & 2,71 \\
\hline Vochysia elliptica (Spr.) Mart. & & & & & 1,53 & 0,74 & 2,50 & 1,59 \\
\hline Vochysia sp. & & & & & 0,76 & 5,90 & 2,50 & 3,05 \\
\hline Total & 100 & 100 & 100 & 100 & 100 & 100 & 100 & 100 \\
\hline
\end{tabular}


Tabela 3. Variáveis da estrutura fisionômica para a área total e cada um dos ambientes distintos no trecho de cerrado stricto sensu do PEB em Diamantina-MG.

Table 3. Physiognomic structure variables for the total area and each of the distinct environments in the cerrado stricto sensu stretch in PEB, Diamantina, MG.

\begin{tabular}{lcccc}
\multicolumn{1}{c}{ Variáveis } & Total & Ambiente 1 & Ambiente 2 & p-valor \\
Número de indivíduos & 445 & 183 & 262 & 0,9094 \\
Densidade (ind/ha) & 445 & 457,5 & 426,7 & 0,9094 \\
\hline Área Basal $\left(\mathrm{m}^{2} / \mathrm{ha}\right)$ & 6,1 & 5,8 & 6,2 & 0,8531 \\
HT média $(\mathrm{m})$ & 4,29 & 4,85 & 3,90 & 0,6448 \\
DAS médio $(\mathrm{cm})$ & 11,7 & 12,0 & 11,2 & 0,1502 \\
\hline
\end{tabular}

p-valor: teste $t$ para amostras independentes.

$16 \mathrm{~m}$ e tronco de 40 a $70 \mathrm{~cm}$ de diâmetro (Lorenzi, 1992).

Os microambientes existentes no cerrado stricto sensu com afloramentos rochosos propiciam e facilitam o estabelecimento de espécies de formações florestais (Moura, 2010). Isso ocorre devido à presença de reentrâncias e fendas nos afloramentos rochosos, que propiciam o acúmulo de água e tornam favorável o estabelecimento de espécies associadas às matas de galeria. Outro fator pode ser o acúmulo de serrapilheira (matéria orgânica) nas fendas e degraus das rochas, o que pode ocasionar o estabelecimento de espécies de solos mais férteis, como nas florestas (Felfili \& Fagg, 2007).

Embora o conjunto de espécies de maior VI, ou seja, aquelas que dominam a vegetação, seja distinto nos ambientes, não houve diferença significativa $(\mathrm{p}<0,05)$ entre os parâmetros estruturais avaliados em cada um deles. A Tabela 3 apresenta os valores para os seguintes parâmetros: número de indivíduos, densidade, área basal, altura média e diâmetro médio, e os respectivos valores de $\mathrm{p}$.

Este resultado corrobora o encontrado por Abreu et al. (2012), em que, apesar de encontrarem menores densidades no cerrado com afloramentos rochosos, os valores de área basal e de altura dos indivíduos não diferiram entre o cerrado típico e o cerrado com afloramentos rochosos. No entanto, o resultado deste trabalho contraria a sugestão de Kurtz \& Araújo (2000) de que os afloramentos rochosos podem limitar o porte dos indivíduos lenhosos, assim como contraria a afirmação de Ribeiro \& Walter (2008) de que, em trechos de cerrado com afloramentos rochosos, a densidade de indivíduos é variável e dependente da quantidade de rochas.

\section{CONCLUSÃO}

A presença dos afloramentos rochosos seleciona um conjunto de espécies específicas e diferentes daquele conjunto de espécies do ambiente sem os afloramentos. Espécies distintas e com características ecológicas dessemelhantes são indicadoras de cada um dos ambientes. Com relação à estrutura da comunidade lenhosa, a presença dos afloramentos rochosos não acarreta diferença significativa nos parâmetros estruturais: número de indivíduos, diversidade, área basal, altura média e diâmetro médio.

\section{AGRADECIMENTOS}

Ao projeto 'Desenvolvimento de tecnologias para produção sustentada de três espécies do cerrado para o Alto Jequitinhonha, Minas Gerais', do Departamento de Engenharia Florestal da Universidade Federal dos Vales do Jequitinhonha e Mucuri - UFVJM, e aos seus financiadores: Fundação de Amparo à Pesquisa do Estado de Minas Gerais - FAPEMIG, Instituto Estadual de Florestas - IEF, Secretaria de Estado de Ciência, Tecnologia e Ensino Superior de Minas Gerais - SECTES e Fundação Diamantinense de Apoio ao Ensino, Pesquisa e Extensão - FUNDAEPE, pela oportunidade de realizar a coleta dos dados deste trabalho.

\section{STATUS DA SUBMISSÃO}

Recebido: 11/09/2013

Aceito: 10/12/2013

Publicado: 31/02/2014 


\section{AUTOR(ES) PARA CORRESPONDÊNCIA}

\section{Sílvia da Luz Lima Mota}

Departamento de Engenharia Florestal, Universidade de Brasília - UnB, CEP 70910-900, Brasília, DF, Brasil

e-mail: silvialimamota@gmail.com

\section{REFERENNCIAS}

Abreu MF, Pinto JRR, Maracahipes L, Gomes L, Oliveira $\mathrm{EA}$, Marimon BS et al. Influence of edaphic variables on the floristic composition and structure of the tree-shrub vegetation in typical and rocky outcrop cerrado areas in Serra Negra, Goiás State, Brazil. Brazilian Journal of Botany 2012; 35: 259-272. http://dx.doi.org/10.1590/ S1806-99592012000300005

Amaral AG, Pereira FFO, Munhoz CBR. Fitossociologia de uma área de cerrado rupestre na fazenda sucupira, Brasília-DF. Cerne 2006; 12(4): 350-359.

Angiosperm Phylogeny Group - APG III. An update of the Angiosperm Phylogeny Group classification for the orders and families of flowering plants: APG III. Botanical Journal of the Linnean Society 2009; 161: 105-121. http://dx.doi.org/10.1111/j.10958339.2009.00996.x

Dufrêne M, Legendre P. Species assemblages and indicator species: the need for a flexible asymmetrical approah. Ecological Monograhs 1997; 67: 345-366.

Felfili JM, Silva-Junior MC, Sevilha AC, Fagg CW, Walter BMT, Nogueira PE, et al. Diversity, floristic and structural patterns of cerrado vegetation in Central Brazil. Plant Ecology 2004; 175: 37-46. http://dx.doi. org/10.1023/B:VEGE.0000048090.07022.02

Felfili JM, Silva-Junior MC. Diversidade alfa e beta no cerrado sensu stricto, Distrito Federal, Goiás, Minas Gerais e Bahia. In: Scariot A, Sousa-Silva JC, Felfili JM, organizadores. Cerrado: Ecologia, Biodiversidade e Conservação. Brasília: Ministério do Meio Ambiente; 2005. p. 143-154.

Felfili JM, Fagg CW. Floristic composition, diversity and structure of the "cerrado" sentido restrito on rocky soils in northern Goiás and southern Tocantins, Brazil. Revista Brasileira de Botânica 2007; 30(3): 375-385.

Fujaco MAG, Leite MGP, Pontes SR, Ornelas AR. Controle geomorfológico e antrópico na distribuição de candeias (Eremanthus sp.) no parque estadual do Itacolomi, Minas Gerais. In: VI Simpósio Nacional de Geomorfologia; 2006. Goiânia.

Goodland RA. Análise ecológica da vegetação do cerrado. In: Goodland RA, Ferri MG. Ecologia do Cerrado. São Paulo: USP; 1979. p. 61-171.
Haridasan M. Nutrição mineral de plantas nativas do cerrado. Revista Brasileira de Fisiologia Vegetal 2000; 12(1): 54-64.

Kent M, Coker P. Vegetation description and analysis; a practical approach. London: Bealhaven Press; 1992. p. 363 .

Kurtz BC, Araújo DSD. Composição florística e estrutura do componente arbóreo de um trecho de Mata Atlântica na Estação Ecológica Estadual do Paraíso, Cachoeiras de Macacu, Rio de Janeiro, Brasil. Rodriguésia 2000; 51: 78-115.

Lima TA, Pinto JRR, Lenza E, Pinto AS. Florística e estrutura da vegetação arbustivo-arbórea em uma área de cerrado rupestre no Parque Estadual da Serra de Caldas Novas, Goiás. Biota Neotrópica 2010; 10(2). http://dx.doi.org/10.1590/S1676-06032010000200020

Lorenzi H. Árvores brasileiras: Manual de identificação e cultivo de plantas arbóreas nativas do Brasil. São Paulo: Nova Odessa, Editora Plantarum 1(1); 1992.

Lorenzi H. Árvores brasileiras. Manual de identificação e cultivo de plantas arbóreas nativas do Brasil. São Paulo: Nova Odessa, Editora Plantarum 1(2); 1998.

Lorenzi H. Árvores brasileiras. Manual de identificação e cultivo de plantas arbóreas nativas do Brasil. São Paulo: Nova Odessa, Editora Plantarum 2(2); 2002.

McCune B, Mefford MJ. Multivariate analysis of ecological data. Gleneden Beach: MjM Software; 1999.

Miranda SC, Silva-Junior MC, Salles LA. A comunidade lenhosa de cerrado rupestre da Serra Dourada, Goiás. Heringeriana 2007; 1: 43-53.

Moura IO, Klein VLG, Felfili JF, Ferreira HD. Diversidade e estrutura comunitária de cerrado sentido restrito em afloramentos rochosos no Parque Estadual dos Pireneus, Goiás. Revista Brasileira de Botânica 2010; 33(3): 55-467.

Moura IO. Fitogeografia do cerrado rupestre: relações florístico-estruturais e ecológicas de espécies lenhosas [tese]. Brasília: Faculdade de Ecologia, Universidade de Brasília; 2010.

Mueller-Dombois D, Ellenberg H. Aims and methods of vegetation ecology. New York: John Wiley \& Sons; 1974. $547 \mathrm{p}$.

Neves SC, Abreu PAA, Fraga LMS. Fisiografia. In: Silva AC, Pedreira LCVSF, Abreu PAA, editores. Serra do Espinhaço Meridional: Paisagens e Ambientes. Belo Horizonte: O Lutador; 2005. p. 47-58.

Pinto JRR, Lenza E, Pinto AS. Composição florística e estrutura da vegetação arbustivo-arbórea em um cerrado rupestre, Cocalzinho de Goiás, Goiás. Revista Brasileira de Botânica 2009; 32(1): 1-10.

$\mathrm{R}$ Core Team. A language and environment for statistical computing. Vienna: R Foundation for Statistical 
Computing; 2012. Available from: http://www.Rproject.org/.

Ratter JA, Bridgewater S, Ribeiro JF. Analysis of the floristic composition of the Brazilian cerrado vegetation III: comparison of the woody vegetation of 376 areas. Edinburgh Journal of Botany 2003; 60: 57-109. http:// dx.doi.org/10.1017/S0960428603000064

Reatto A, Correia JR, Spera ST, Martins ES. Solos do Bioma do Cerrado: aspectos pedológicos. In: Sano SM, Almeida SP, editores. Cerrado: ecologia e flora. Planaltina: Embrapa Cerrados; 2008. v. 1, p. 151-199.

Ribeiro JF, Walter BMT. Fitofisionomias do Cerrado. In: Sano S, Almeida S, editores. Cerrado: ambiente e flora. Planaltina: Embrapa CPAC; 2008. v. 1, p. 89-166.

Scarano FR. Perspectives on biodiversity science in Brazil. Scientia Agricola 2007; (64): 439-447. http:// dx.doi.org/10.1590/S0103-90162007000400016

Scolforo JRS, Oliveira AD, Davide AC, Mello JM, Acerbi Junior FW. Manejo sustentável da candeia Eremanthus erythropappus e Eremanthus incanus. Lavras: UFLAFAEPE; 2002. Relatório técnico científico, 350 p.

Silva-Junior MC. 100 Árvores do Cerrado: guia de campo. Brasília: Distrito Federal, Rede de Sementes do Cerrado; 2005.

Silva-Junior MC, Pereira BAS. + 100 árvores do cerrado - Matas de Galeria: guia de campo. Brasília: Distrito Federal, Rede de Sementes do Cerrado; 2009.

Silva-Junior MC. 100 árvores do cerrado - sentido restrito: guia de campo. Brasília: Distrito Federal, Rede de Sementes do Cerrado; 2012.

Ter Braak CJF. Ordination. In: Jongman RHG, ter Braak CJF, Van Tongeren OFR, editors. Data analysis in community and landscape ecology. Cambridge: Cambridge University Press; 1995. p. 91-173. http:// dx.doi.org/10.1017/CBO9780511525575.007

Ter Braak CJF. The analysis of vegetation-environment relationships by canonical correspondence analysis. Vegetation. 1987. 